\title{
Control and Simulation of Torque Converter Lock-up Clutch
}

\author{
Li-mei Zhao ${ }^{1,2}$, Huai-chao $\mathrm{Wu}^{2}$, Zheng-hu Peng ${ }^{2}$, Cheng Zhang ${ }^{2}$, Shi-hao Tang ${ }^{1}$, Yun-xiang Long ${ }^{3}$ and Guo-qiao Li ${ }^{3}$ \\ ${ }^{1}$ Key Laboratory of Advanced Manufacturing Technology of Ministry of Education, Guizhou University, China \\ ${ }^{2}$ College of Mechanical Engineering, Guizhou University, Guiyang, China \\ ${ }^{3}$ Guizhou Winstar Hydraulic Transmission Machinery Co., Ltd, Zunyi, China
}

\begin{abstract}
Considering the requirements of dynamic performance and quality during the torque converter locking process, a two parameters latch-up control strategy was designed, and a simulation method to study the control performance by use of processor in loop (PIL) simulation technology was proposed. Through the study of the practically applied requirements of a loader, a two parameter lock-up control strategy with turbine speed signal and gear signal as main control parameters was developed. According to the control strategy, the logic diagram of real-time locking and unlocking control of the latch-up clutch was established by using Matlab/Simulink. Then, PIL technology was used to realize the co-simulation of CCS and Simulink. Simulation results show that the latch-up control model is correct and the lock-up clutch can effectively lock and unlock in accordance with the lock-uprule.
\end{abstract}

\section{Introduction}

The application of torque converter in vehicles greatly simplifies the vehicle handling, improves ride comfort, safety, crossing country and average vehicle speed, but the biggest defect of hydraulic transmission is inefficiency. In order to reduce the fuel consumption of torque converter, a controllable latch-up clutch is installed between the pump wheel and the turbine of the torque converter. When the driving condition of the car reaches the setting target, the lock-up clutch is controlled to lock, the pump wheel is integral to the turbine, and the torque converter then turns into mechanical transmission. To a certain extent, it relieves the problem of low transmission efficiency.

Under the condition that the converter structure type and parameters have been determined, control of lock-up clutch is mainly focus on the improvement of lock-up control quality and the rationality of lock-up rule. The main purpose of lock-up control is to reduce the lock-up impact and improve vehicle dynamic performance. The quality of lock-up process mainly depends on the control during engagement process. In recent years, many domestic scholars have done a great deal of research work in optimizing the lock-up points, realizing multi parameter lock-up control, and control methods such as buffer control by manipulating oil pressure[1-4].In this study, the lock-uprule of the lock-up clutch of a loader was studied, and the simulation model of the lock-up control was established, and the co-simulation of CCS and Simulink analysis was realized by using PIL detection technology. 


\section{Lock-up control law of the torque converter}

\subsection{The rule of latch-up control}

The rule of latch-up control is generally divided into single parameter control and two parameters control. And two-parameter control is a control strategy with two signals as the main control signals and the rest for the auxiliary control signals. In this study, loaders generally work on sites where the environments are severe, so two-parameter control was chosen to reduce the impact of the environment on the equipment. In the two-parameter control system, the turbine speed signal and the gear signal were chosen as the main control parameters and the steering signal, the throttle signal and the braking signal were chosen as the auxiliary control parameters.

The clutch control can be divided into dynamic locking, economic locking, steering state locking, braking state locking and integrated locking. And the rule of integrated locking has good power performance and fuel economy as well as the operability of the vehicle, and it also ensures great probability for the integrated locking performance of the vehicle for different applications. Therefore, the integrated locking rule was chosen to research on the control system. The integrated locking rule designed of the loader was as follows:

1)Whether the clutch would lock or not is determined by the turbine speed in the braking state;

2)In the steering condition, the unlocking of the latch-up clutch is determined by throttle angle, and the latch-up clutch is not locked when the throttle angle is less than $20 \%$;

3)Locking would prevent the shift from running smoothly when shifting, so the lock-up clutch needs to be unlocked;

4)Whether the latch-up clutch would lock or not can be judged by the rotation speed of the turbine when not to change gear;

5)According to different gears and using purposes, at the first gear, the clutch must be unlocked in order to obtain greater torque for starting, climbing, and high power; at the second gear, the locking is determined by the throttle angle. And when the throttle angle is less than $20 \%$, the clutch is unlocked in order to obtain greater torque; when the throttle angle is more than $20 \%$, whether the clutch would lock or not is judged by the rotation speed of the turbine and the throttle angle in order to make the vehicle more efficient; At the third gear, the clutch needs to be locked in order to make the vehicle get higher speed and larger utilization of power.

\subsection{Determination of lock-up point of the lock-up clutch}

In order to ensure the dynamic performance of the vehicle, double parameters (turbine speed-throttle angle) lock-up rule is usually adopted to select the lock-up points. Under a certain throttle angle, the intersection of two turbine shaft output torque curves under hydraulic conditions (unlocked) and mechanical conditions (locked) respectively is selected, the turbine speed of the intersection point is the lock-up point at the throttle angle. The torque of the torque converter is the output torque of the pump wheel. It can be expressed by equation (1)[5-6]

$$
M_{B}=\lambda_{B} m_{B}^{2} D^{5}
$$

Where $\mathrm{D}$ is the effective diameter of the circulating circle of torque converter, $\mathrm{D}=0.397 \mathrm{~m} ; \lambda \mathrm{B}$ is the torque factor; $\gamma$ is the working liquid density $(\mathrm{kg} / \mathrm{m} 3), \mathrm{nBis}$ the pump wheel speed $(\mathrm{r} / \mathrm{min})$.

The relationship between the torques and rotational speeds of turbine and pump wheel are shown as equation (2), (3).

$$
\begin{gathered}
M_{\mathrm{Ti}}=K_{\mathrm{i}} M_{\mathrm{Bi}}(2) \\
n_{\mathrm{Ti}}=i n_{\mathrm{Bi}}
\end{gathered}
$$

Where $M_{\mathrm{T}}$ is the turbine torque $(\mathrm{N} \cdot \mathrm{m}) ; K$ is the torque coefficient; $M_{\mathrm{B}}$ is the pump torque $(\mathrm{N} \cdot \mathrm{m}) ; n_{\mathrm{T}}$ is the turbine output speed ( $\mathrm{r} / \mathrm{min}) ; n_{\mathrm{Bi}}$ is the pump wheel speed $(\mathrm{r} / \mathrm{min}) ; i$ is the speed ratio.

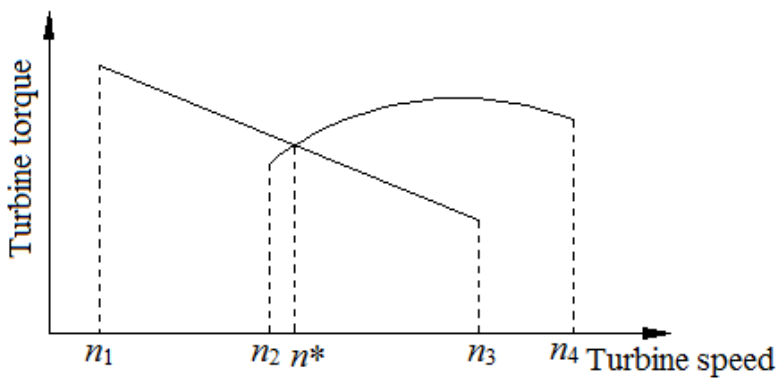

Figure 1. Schematic diagram of double-parameter power lock-up point design

At a certain throttle angle, according to the original data of the engine and formula equation (1), (2), (3), the 
output curve of the turbine shaft under hydraulic conditions (unlocked) and mechanical conditions (locked)can be calculated under a certain throttle angle. The design of the lock-up point diagram is shown as Figure 1, where $n *$ is the lock-up point.

\section{Modeling and Simulation of torque converter lock-up control}

According to the lock-up control rule established, the DSP control system of lock-up clutch was simulated and analyzed by adopting the concept of model design. By using CCS and Simulink combined simulation method, the control flow chart was built according to the lock-up rule in Simulink/Stateflow. Then, TI compiler (Mainly CCSIDE integrated development environment) oriented code was generated by Matlab Real-Time-Workshio (RTW). The code compile, link and download were completed, then run the program on the TMS320F28335 development board to realize the simulation system[7-13].

\subsection{Establishment of latch-up control model}

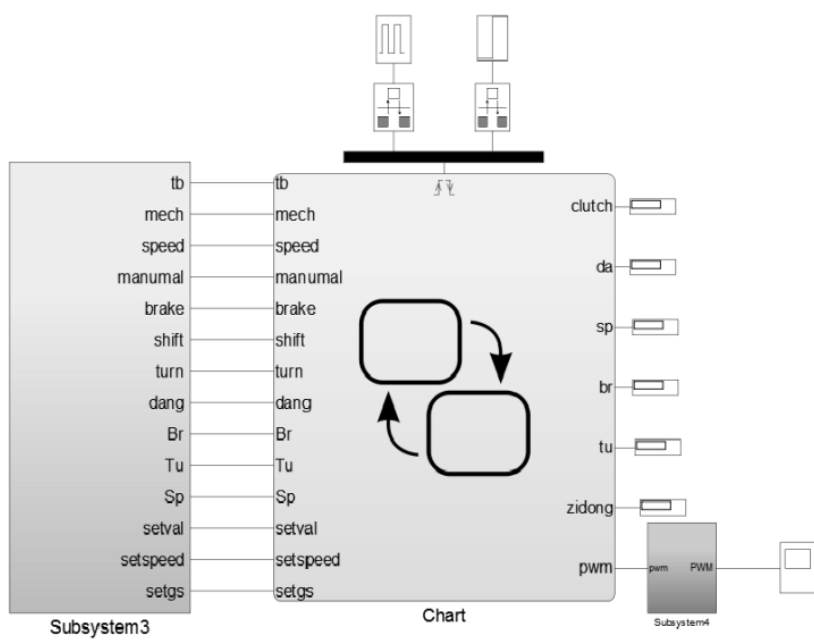

Figure 2. Simulation model of lock-up clutch control

The model of the latch-up control system established in Simulink is shown in Figure 2. Signals such as automatic / manual switch signal, turbine speed signal, shift signal, brake signal, speed signal and turn signal were simulated in Simulink and fed into the lock-up control system in Stateflow. In these signals, the shift signal and the turbine speed signal are the main control signals, while the rest are auxiliary signals. These signals can be set were encapsulated into subsystem Subsystem3. The signals were fed into the logical control in
Stateflow/chart, then, according to the signals, the right PWM wave is output by logical judgment. In the output signals, the Clutch of 1 means the latching state and the Clutch of 0 means the unlock status. The Zidong of 1 represents the braking state; the Zidong of 0 represents the manual state. The $\mathrm{Da}, \mathrm{SP}, \mathrm{Br}$, and Tu respectively indicate the initial gear position, speed, braking and turning signals of the vehicle. PWM is the final output duty cycle signal. It acts directly on the latch-up clutch signal valve in the hydraulic system and realizes the control of the combination cavity and the release cavity oil pressure, thus realizes the locking and unlocking control of the clutch.

The Stateflow logic control flow chart inside the Chart is shown in Figure 3.

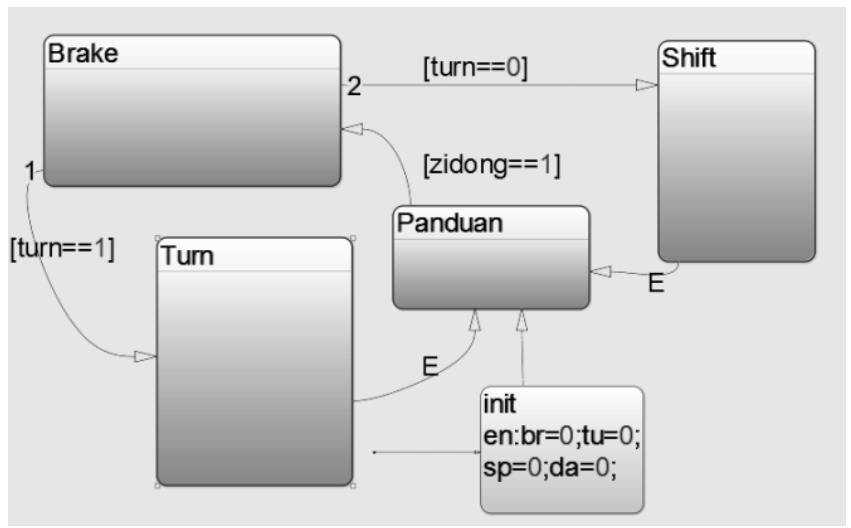

Figure 3. Stateflow logic control flow chart

At the beginning of the operation, the program was initialized in the init state. Then, shift, speed, brake and turn signals were cleared. After the initialization processing, the signal get into the judgment module (Panduan). When the signal Zidong= 1the state shifted, whether the program entered into the next cycle was determined by other signals. If Zidong $=0$, it was always in the Panduan state, executed the Panduan module repeatedly. If the condition[zidong==1] was satisfied, the program was transferred to the braking state and the brake logic control was performed. When signal brake $=0$, the turn signal was judged. In chart, when the signal turn=1, program would execute steering logic control. If the signal turn $=0$, the shift signal was judged and the program would perform the program under the condition of a shift signal, or a program without a shift signal. 


\subsection{The hardware of DSP control simulation system}

Hardware is mainly ZQ28335 board and XDS100USB emulator developed by Hefei 00 electronic as shown in Figure 4. In the development board, digital tubes were used to display changes in gears, speed, and other signals. The blocking value and gear changes, etc. were set by the matrix keyboard in the development board. The emulator was used to connect the development board and CCS software to realize the co-simulation of hardware and software. First, the environment variables that Simulink and CCS match were set up in the Windows system.Then, the models those consistent with the development board and emulator were selected, and the simulation model was built in Simulink. The interfaces of the chip used in the simulation model were set. Finally, the model in the Simulink was compiled into the development board by emulator, and the co-simulation of the lock-up control system with Simulink and CCS was realized.

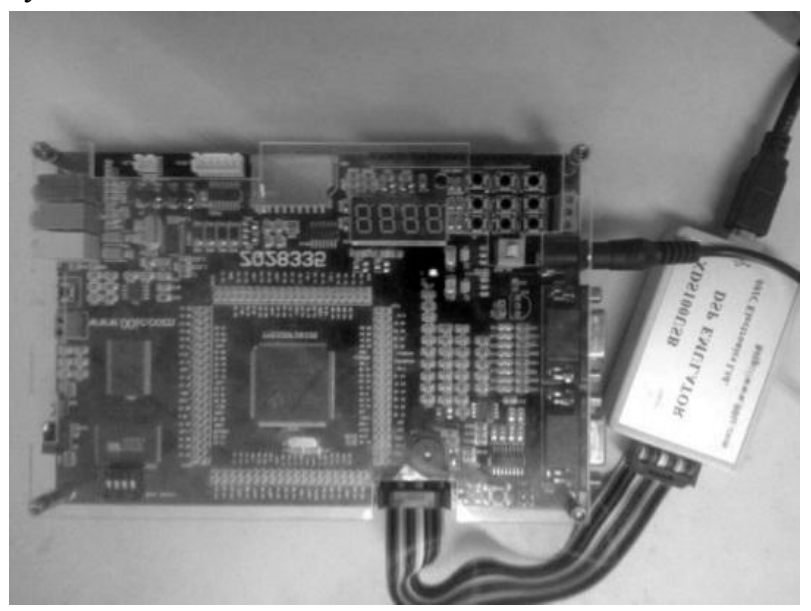

Figure 4.Development board and emulator

\subsection{Analysis of simulation results}

The signal brake $=0$ and signal turn $=0$ were set, and the transmission was placed in first gear, these signals detected by the PIL processor in the loop. The simulation output of the PWM wave is as shown in Figure 5.By changing the duty cycle of PWM wave in real time, the high speed on-off valve in hydraulic system can be controlled, so that the lock-up clutch can be controlled indirectly. As shown in Figure 5, the duty cycle of the output PWM wave is very small. So that the high-speed on-off valve in the hydraulic control system controlled by the PWM wave was off, lock - up clutch was controlled lock. The simulation result satisfied the lock control law.In the former 30s, the amplitude of the wave is zero because the flow chart designed leaves sufficient time for each signal to occur, and also because each trigger signal has a time delay during its production

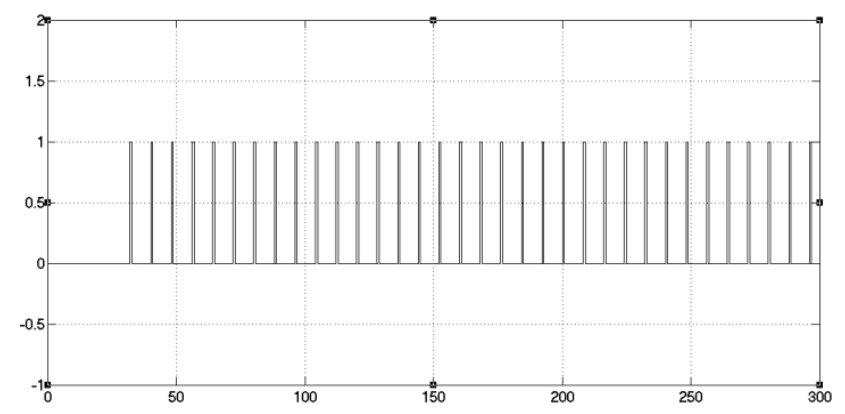

Figure5. The simulation output PWM wave in first gear

In the simulation process, the transmission was set to second gear, throttle angle and turbine speed were changed. In order to facilitate observation of changes of output PWM wave when the locking condition was satisfied and the locking condition was not satisfied in second gear, in the former 150s the throttle angle was set to $35 \%$, the turbine speed was set to $2000 \mathrm{rpm}$, which satisfied the lock condition, and 150 seconds later set throttle angle was set to $15 \%$, the turbine speed was set to 1000rpm, which did not satisfy the locking conditions. These signals detected by the PIL processor in the loop. The simulation output of the PWM wave is as shown in Figure 6. As shown in Figure 6, the former 30sis the transition time for program execution. From $30 \mathrm{~s}$ to $150 \mathrm{~s}$, the throttle angle and the turbine speed meet the locking conditions, the duty cycle of the output PWM is $70 \%$.And the PWM would control the clutch locked. 150s later the throttle angle and the turbine speed do not meet the locking conditions, the duty cycle of the output PWM is $30 \%$. And the PWM would control the clutch unlocked. From the above analysis, it can be seen that the simulation results meet the control requirements of the lockup clutch.

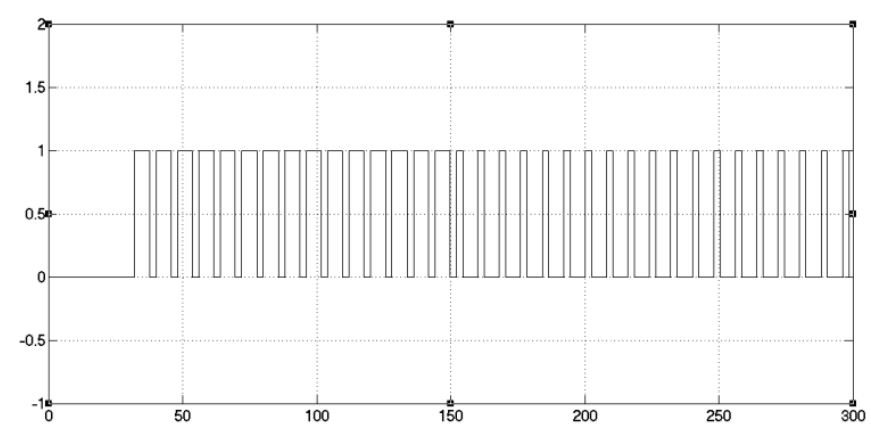

Figure 6.The simulation output PWM wave in second gear 


\section{Conclusions}

According to the established control rule of lock-up clutch of torque converter, the simulation system of lock-up process of lock-up clutch was built by the method of co-simulation of MATLAB/Simulink and CCS, the lock-up processes under the conditions of different gears were simulated, and the simulation results were analyzed. The results show that the established control ruleis reasonable and meet the engineering requirements. It is proved that the PIL simulation method presented in this paper is feasible and effective.This study presents a feasible scheme for the ECU development and the design of other similar control systems.

\section{Acknowledgements}

This project is supported by training plan for high-level innovative talent in Guizhou Province (Grant No. Q. K. H. P.T. R. C[2016]5659), the science and technology major project of Guizhou Province in China (Grant No. Q. K. H. Z. D. Z. X. Z [2013]6015) and the introduction of talent fund of Guizhou University in China (Grant No.G. D. R. J. H. Z[2014]41).

\section{References}

1. B. Long, SCI. \& TEC. INFO. 34, 37(2007)

2. J.Hu, D.Qin, X.Jing, J. of Chongqing Univ.27,1(2004)

3. S.Yang, H. Yuand J.Liu, J. OF MECH. ENG.50, 152(2014)

4. X.Jiang and D. Qin, Chongqing Univ.22(2014)

5. Z. Hang, S. Zhao and Q. Li, ShanghaiAUTO. 4, 13(2000)

6. L.Yan and H.Huang, Jiling Univ. 15(2005)

7. C.Liu and W. Zhang, Shandong Univ. of Tech. 20(2009)

8. Z.Song and B.Zhang, Hefei Univ. of Tech. 12(2010)

9. D.Zhang and A.Ge, Jilin Univ. 30(2005)

10. Z.Li and Y.Lei, Jilin Univ. 23(2014)

11. E.Hans, Thies Dr, Arthur Wetzel. Drive System Tech.4,1(1998)

12. Kato Yoshiaki, Akasaka Ryokoh and Yamazaki Tsutomu.J.of Japan. Soc. of Trib.42,485 (1997)

13. Q.Chen, D.Qin and X.Ye, J. of Sys. SL.22,669(2010) 